\title{
Low-resolution pressure reactivity index and its derived optimal cerebral perfusion pressure in adult traumatic brain injury: a CENTER-TBI study
}

Lennart Riemann ${ }^{1,2}$, Erta Beqiri ${ }^{2,3}$, Peter Smielewski², Marek Czosnyka², Nino Stocchetti ${ }^{3,4}$, Oliver Sakowitz ${ }^{5}$, Klaus Zweckberger ${ }^{1}$, Andreas Unterberg ${ }^{1}$, Alexander Younsi ${ }^{1^{*}}$ (D) and the CENTER-TBI High Resolution ICU (HR ICU) Sub-Study Participants and Investigators

\begin{abstract}
Background: After traumatic brain injury (TBI), brain tissue can be further damaged when cerebral autoregulation is impaired. Managing cerebral perfusion pressure (CPP) according to computed "optimal CPP" values based on cerebrovascular reactivity indices might contribute to preventing such secondary injuries. In this study, we examined the discriminative value of a low-resolution long pressure reactivity index (LPRx) and its derived "optimal $\mathrm{CPP}^{\prime \prime}$ in comparison to the well-established high-resolution pressure reactivity index (PRx).

Methods: Using the Collaborative European NeuroTrauma Effectiveness Research in Traumatic Brain Injury (CENTERTBI) study dataset, the association of LPRx (correlation between 1-min averages of intracranial pressure and arterial blood pressure over a moving time frame of $20 \mathrm{~min}$ ) and PRx (correlation between 10-s averages of intracranial pressure and arterial blood pressure over a moving time frame of $5 \mathrm{~min}$ ) to outcome was assessed and compared using univariate and multivariate regression analysis. "Optimal CPP" values were calculated using a multi-window algorithm that was based on either LPRx or PRx, and their discriminative ability was compared.

Results: LPRx and PRx were both significant predictors of mortality in univariate and multivariate regression analysis, but PRx displayed a higher discriminative ability. Similarly, deviations of actual CPP from "optimal CPP" values calculated from each index were significantly associated with outcome in univariate and multivariate analysis. "Optimal CPP" based on PRx, however, trended towards more precise predictions.

Conclusions: LPRx and its derived "optimal CPP" which are based on low-resolution data were significantly associated with outcome after TBI. However, they did not reach the discriminative ability of the high-resolution PRx and its derived "optimal CPP." Nevertheless, LPRx might still be an interesting tool to assess cerebrovascular reactivity in centers without high-resolution signal monitoring.

(Continued on next page)
\end{abstract}

\footnotetext{
* Correspondence: alexander.younsi@med.uni-heidelberg.de

'Department of Neurosurgery, Heidelberg University Hospital, INF 400, 69120 Heidelberg, Germany

Full list of author information is available at the end of the article
}

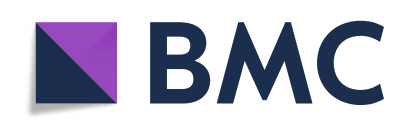

() The Author(s). 2020 Open Access This article is licensed under a Creative Commons Attribution 4.0 International License, which permits use, sharing, adaptation, distribution and reproduction in any medium or format, as long as you give appropriate credit to the original author(s) and the source, provide a link to the Creative Commons licence, and indicate if changes were made. The images or other third party material in this article are included in the article's Creative Commons licence, unless indicated otherwise in a credit line to the material. If material is not included in the article's Creative Commons licence and your intended use is not permitted by statutory regulation or exceeds the permitted use, you will need to obtain permission directly from the copyright holder. To view a copy of this licence, visit http://creativecommons.org/licenses/by/4.0/. The Creative Commons Public Domain Dedication waiver (http://creativecommons.org/publicdomain/zero/1.0/) applies to the data made available in this article, unless otherwise stated in a credit line to the data. 
(Continued from previous page)

Trial registration: ClinicalTrials.gov Identifier: NCT02210221. First submitted July 29, 2014. First posted August 6, 2014.

Keywords: Cerebral autoregulation, Cerebrovascular reactivity, Cerebral perfusion pressure, CPPopt, Traumatic brain injury

\section{Background}

Following severe traumatic brain injury (TBI), secondary injury cascades occur that affect cerebral blood flow (CBF). They may lead to ischemia when the cerebral perfusion pressure (CPP), the pressure gradient for cerebral blood flow defined as arterial blood pressure (ABP) minus intracranial pressure (ICP), is too low or to hyperemia and increased ICP when the CPP is too high [1-3]. The brain is vulnerable to changes in CPP after severe TBI because cerebral autoregulation, which normally maintains constant $\mathrm{CBF}$ during changes in ABP, is often impaired in those patients [1,3-5]. A mainstay in the clinical management of TBI is therefore the avoidance of secondary brain injury by controlling ICP and ensuring adequate, non-harmful CBF by regulating CPP. Current guidelines (2016) by the Brain Trauma Foundation recommend keeping CPP between 60 and $70 \mathrm{mmHg}$ [6]. However, likely due to the heterogeneity of cerebral injuries in patients with TBI, a CPP-oriented therapy with one fixed target for all patients failed to demonstrate improved neurological outcome compared to ICPtargeted therapy in a large randomized-controlled trial [7]. This is why a patient-customized approach has been proposed which uses the pressure reactivity index (PRx) to determine the optimal CPP (CPPopt) in an individual patient. The PRx, calculated as a moving correlation coefficient between slow waves of ABP and ICP, is a surrogate marker for cerebral autoregulation and has been associated with outcome after TBI in multiple studies [8-12]. Positive PRx values indicate dysfunctional cerebrovascular reactivity and are associated with increased mortality and unfavorable outcome, while negative values indicate intact pressure reactivity [9]. Using computational methods, this relationship can be exploited to determine an optimal CPP that corresponds to the lowest, most favorable PRx values in a patient [12, 13]. As the PRx and thus CPPopt are derived from ABP and ICP signals that are continuously monitored, the CPPopt recommendation can be constantly updated and refined, thereby providing the possibility to customize the clinical management also over the course of time in an individual patient. The automated CPPopt algorithm introduced by Aries et al. which uses a single, 4-h moving monitoring window to calculate CPPopt has been developed further to a multi-window algorithm.
Deviations of CPP from CPPopt have been shown to correlate with clinical outcome in several retrospective studies [13-15], and the first prospective study assessing the feasibility of clinical management based on continuous determination of CPPopt is currently ongoing (COGiTATE trial) [16]. However, as the PRx and thus PRx-based CPPopt calculations require continuous, fullresolution waveforms of $\mathrm{ABP}$ and $\mathrm{ICP}$, the CPPopt concept is currently limited to specialized neurocritical care units. In an attempt to increase accessibility of the PRx concept, a similarly calculated $\mathrm{PRx}$ variant called the long pressure reactivity index (LPRx) has been introduced which can be derived from lower resolution, minute-by-minute resampled ICP and ABP signals that standard monitoring devices in most intensive care units (ICU) can provide [17]. However, it remains unclear whether minute-by-minute monitoring might be of high enough resolution to evaluate autoregulation in patients with TBI and derive clinically relevant information from it. In fact, previous studies have yielded mixed results and drawn different conclusions [8, 17-19].

In light of these previous results, we assessed the discriminative value of LPRx and PRx and the performance of the most recent, multi-window CPPopt algorithm currently used in the COGiTATE trial but built on the LPRx instead of the PRx.

\section{Methods \\ Patient cohort}

For this study, all patients from the high-resolution (HR) ICU cohort of the Collaborative European NeuroTrauma Effectiveness Research in TBI (CENTER-TBI) study (EC grant 602150), prospectively recruited between January 2015 and December 2017 at 21 centers in the European Union (EU), were screened and included if they met the following inclusion criteria: (a) availability of highfrequency ICU monitoring data (i.e., continuous ABP and ICP monitoring), (b) availability of Glasgow Outcome Scale Extended (GOSE) at 6 months, (c) age of 18 years or older, and (d) ICP was measured via intraparenchymal probe. During the analysis, 8 patients were excluded from our study because PRx- or LPRx-based CPPopt could not be calculated due to either too short or interrupted monitoring data after artifact removal (3 patients) or very high mean ICP values over the entire 
monitoring period exceeding $55 \mathrm{mmHg}$ and resulting in persistent PRx values close to +1 , making the CPPopt calculation meaningless (5 patients). For all remaining patients, the following demographic data was retrieved from the CENTER-TBI Neurobot database (version 2.0, CENTER core): age, sex, Glasgow Coma Scale (GCS), GCS-motor component, pupillary response at admission (bilaterally reactive, unilaterally reactive, bilaterally unreactive), and necessity of decompressive surgery (yes/no). For assessment of outcome, the imputed 6-month GOSE variable was used which includes both observed ratings and imputed values. For comparative analyses, patients were dichotomized into fatal vs. non-fatal outcome and unfavorable (GOSE 1-4) vs. favorable (GOSE 5-8) outcome.

\section{ICU monitoring data: recording and processing}

High-frequency digital signals from continuous ABP and ICP monitoring during intensive care treatment were recorded for all patients included in this study. Recording was intended to start within $24 \mathrm{~h}$ of injury and encompassed the entire time that ICP/ABP monitoring was clinically required. $\mathrm{ABP}$ was monitored through radial or femoral arterial lines while ICP was monitored through intraparenchymal ICP probes, parenchymal fiber optic pressure sensors, or external ventricular drains. Signals were either digitally recorded or digitalized via an A/D converter (DT9801; Data Translation, USA MA), hereby sampled at frequency of $100 \mathrm{~Hz}$ or higher, using the ICM+ software (Cambridge Enterprise Ltd., UK), a Moberg CNS Monitor (Moberg Research Inc., USA), or a combination of both. Signal artifacts were removed manually and using automated algorithms. Data processing of all monitored signals was conducted using ICM+. Moving averages of $10 \mathrm{~s}$ (updated every $10 \mathrm{~s}$ ) were calculated for $\mathrm{ABP}, \mathrm{ICP}$, and $\mathrm{CPP}(=\mathrm{ABP}-\mathrm{ICP})$. PRx was calculated as a moving Pearson correlation coefficient between $10 \mathrm{~s}$ averaged ICP and ABP values in a moving time window of $5 \mathrm{~min}$. For the LPRx, 1-min averages of $\mathrm{ICP}$ and $\mathrm{ABP}$ were correlated within a moving time window of $20 \mathrm{~min}$. PRx, LPRx, and minute-by-minute averages of $\mathrm{ABP}$ and ICP were updated every minute. CPPopt was calculated using a multi-window, weighted approach. Details on the exact ICM+ settings and calculation methods can be found in the COGiTATE protocol paper by Beqiri et al. [16]. In short, a 5-min median CPP time trend was calculated along the PRx (Fisher transformed to achieve normal distribution) and these PRx values were grouped into CPP bins of $5 \mathrm{mmHg}$ (within $40-120 \mathrm{mmHg}$ range). The CPP value corresponding to the lowest associated PRx value was determined using an automatic parabolic curve fitting method. For each time point, this method was conducted for 36 different preceding time windows ranging from 2 to $8 \mathrm{~h}$ (with 10- min increasing steps) in length, resulting in $36 \mathrm{PRx}-\mathrm{CPP}$ plots. Following certain inclusion criteria (e.g., rejecting non-physiological values), those 36 different CPPopt values were combined according to particular heuristic weighting rules, which included higher weights given to more recent time windows and rejection of nonparabolic curves, to contribute to the final CPPopt. CPPopt $_{\text {LPRx }}$ was calculated accordingly, however using the LPRx instead of PRx. CPPopt $\mathrm{PRx}_{\text {a }}$ and CPPopt $_{\mathrm{LPRx}}$ were updated every minute. The difference between median CPP and each computed CPPopt $_{\mathrm{PRx}} / \mathrm{CPPopt}_{\mathrm{LPRx}}$ value was continuously calculated for every minute of the recording period $\left(\triangle C P P o p t L_{P R x}, \Delta\right.$ CPPopt $\left._{L P R x}\right)$. The yield of $\mathrm{CPPopt}_{\mathrm{PRx}} / \mathrm{CPPopt}_{\mathrm{LPRx}}$ was defined and calculated as the count of CPPopt values divided by the count of CPP values across the whole recording period. Values of all monitoring parameters (ABP, ICP, PRx, LPRx, CPPopt $_{\mathrm{PRx}}, \quad \mathrm{CPPopt}_{\mathrm{LPRx}}, \quad \Delta$ CPPopt $_{\mathrm{PRx}}, \quad \Delta$ CPPopt $_{\mathrm{LPRx}}$ ) were at last averaged over the entire monitoring period for each patient.

\section{Statistical analysis}

Demographical variables and ICU monitoring parameters were descriptively analyzed and compared between dichotomized outcome groups using the Mann-Whitney $U$ test for continuous variables and the chi-squared test for categorical variables. Results are given as median + interquartile range (IQR), unless stated otherwise. Spearman's rank correlation coefficient was used to evaluate correlation between PRx and LPRx while a BlandAltman plot was used to assess agreement between both indices. Univariate logistic regression in regard to dichotomized outcomes was applied with either LPRx or PRx as predictors. Discrimination was assessed via area under the receiver operating curve (AUC) and compared using DeLong's test. Multivariate logistic regression was conducted to assess the association between either LPRx or PRx and dichotomized outcomes with adjustment for the IMPACT core variables age, admission GCS motor score (GCS-Motor), and pupil reactivity status (bilaterally reactive, unilateral reactive, bilateral unreactive) [20]. The goodness-of-fit of the full model (IMPACT core variables + LPRx or PRx) was tested against the IMPACT-only model via a likelihood-ratio test, and AUCs were calculated for each model. In all statistical tests, the level of significance was set at 0.05 , and no $p$ value adjustment for multiple testing was applied due to the exploratory nature of this study. All statistical analyses were performed in the R environment [21].

\section{Results}

Patient demographics

A total of 224 patients (176 males and 48 females) ranging in age from 18 to 85 years with a median age of 51 
(IQR, 33-64 years) were included in this study. Patient demographics and mean values of ICU monitoring parameters are provided in Table 1 . The median GCS score at admission was 6 (IQR, 3-10) with a range from 3 to 15 . Decompressive craniectomy was performed in 47 patients during their hospital stay. Six months after admission, 52 patients were dead yielding a mortality rate of $23 \%$. The outcome of 135 patients $(60 \%)$ was considered unfavorable (i.e., GOSE 1-4) at 6 months post-injury.

\section{PRx and LPRx}

Results from Spearman's test showed that there was a significantly positive, albeit only moderate correlation between mean LPRx and PRx values in our patient cohort $(r=0.63, p<0.001$; Fig. 1a). This correlation was slightly higher for the subgroup of patients with severe TBI $(r=0.68, p<0.001)$. A Bland-Altman plot for agreement between LPRx and PRx yielded a mean bias of 0.076 with $95 \%$ limits of agreement at -0.210 and + 0.362 (Fig. 1b). There was a clear association of higher mortality rates with higher mean LPRx and PRx values, as shown in Fig. 2. Accordingly, both LPRx and PRx were significantly higher in patients with fatal outcome compared to surviving patients (LPRx, $0.025(-0.096-$

Table 1 Patient demographics

\begin{tabular}{ll}
\hline & All patients \\
\hline Number of patients & 224 \\
Age (years) & $51(33-64)$ \\
$\quad$ - Female (\%) & $48(21 \%)$ \\
- Male (\%) & $176(79 \%)$ \\
GCS total & $6(3-10)$ \\
GCS motor & $4(1-5)$ \\
Pupillary response & \\
- Both reactive & $150(67 \%)$ \\
- One reactive & $17(8 \%)$ \\
- Both unreactive & $39(17 \%)$ \\
- Unknown & $18(8 \%)$ \\
ABP (mmHg) & $83.6(78.8-90.0)$ \\
ICP (mmHg) & $12.6(9.6-16.1)$ \\
CPP (mmHg) & $71.6(65.2-77.4)$ \\
PRx & $0.027(-0.074-0.162)$ \\
LPRx & $-0.049(-0.145-0.079)$ \\
GOSE & $3(3-5)$ \\
Unfavorable outcome (\%) & $135(60 \%)$ \\
Fatal outcome (\%) & $51(23 \%)$ \\
\hline ABP ateil blood prst
\end{tabular}

$A B P$ arterial blood pressure, $C P P$ cerebral perfusion pressure, GCS Glasgow Coma Scale, GOSE Glasgow Outcome Scale Extended, ICP intracranial pressure, $L P R x$ long pressure reactivity index, $P R x$ pressure reactivity index
$0.212)$ vs. $-0.064(-0.175-0.054), p<0.001 ; \mathrm{PRx}, 0.163$ $(0.016-0.314) \quad$ vs. $0.009 \quad(-0.082-0.112), \quad p<0.001)$. When dichotomizing patients into unfavorable vs. favorable outcome, a significant group difference could only be detected for the PRx but not for the LPRx (PRx, $0.052(-0.067-0.195)$ vs. $-0.014(-0.086-0.098), p=$ 0.013 ; LPRx, $-0.026(-0.142-0.105)$ vs. -0.063 ($0.148-0.051), \quad p=0.204)$. In univariate regression analysis, both PRx and LPRx were significantly associated with mortality (Table 2 (A)), which generally increased with higher index values. Discriminative ability was higher for the PRx (AUC $0.70(0.61-0.79), p<0.001$ ) compared to the LPRx (AUC $0.66(0.58-0.75), p<$ 0.001 ), although this difference did not reach significance in this patient cohort (DeLong's test, $p=0.348$; Fig. 3a). Interestingly, discriminative ability of both reactivity indices seemed to be particularly strong for the subgroup of patients with severe TBI (LPRx: AUC 0.69 (0.57-0.81) and PRx: AUC 0.73 (0.62-0.84)). The discriminative value in regard to unfavorable outcome was considerably worse for both indices, and only the PRx reached significance in univariate regression with an AUC of $0.60(0.52-0.67, p=0.007)$. However, in comparison to the AUC of LPRx (0.55 (0.47-0.63, $p=$ $0.102)$ ), no significant difference could be observed via DeLong's test either $(p=0.167)$.

Similar results were obtained when examining the predictive value of the reactivity indices in relation to patient outcome over the post-traumatic temporal course (Fig. 4a, b). Discrimination tended to be higher for PRx compared to LPRx for both mortality and, with exceptions at days 4 and 5, unfavorable outcome. However, those differences did not reach significance at any observed time point.

When adjusted for age, GCS-Motor, and pupillary response (IMPACT core variables) in multivariate regression analysis, both LPRx and PRx remained independent predictors for mortality (Table 2 (A)). In regard to unfavorable outcome, neither of the indices was found to be a significant predictor in the same multivariate model (PRx: $p=0.120$ and LPRx: $p=0.976$ ). Importantly, when also controlling for ICP and CPP in the multivariate model, only PRx but not LPRx remained significant (Table $2(\mathrm{C})$ ). Nevertheless, adding either LPRx or PRx to the IMPACT core model led to a notable increase of the AUC for prediction of mortality: $0.78(0.70-0.85)$ when adding LPRx and $0.80(0.72-0.88)$ when adding $\mathrm{PRx}$, compared to $0.74(0.66-0.82)$ for the model without indices (Fig. 3b). For both LPRx and PRx, the goodness-of-fit of the combined IMPACT + index model was significantly improved when compared to the mere IMPACT model as assessed by likelihood-ratio tests $\left(\chi^{2}=6.79, p=0.009\right.$ (LPRx), and $\chi^{2}=15.13, p<$ $0.001(\mathrm{PRx})$, respectively). Results and trends in our 

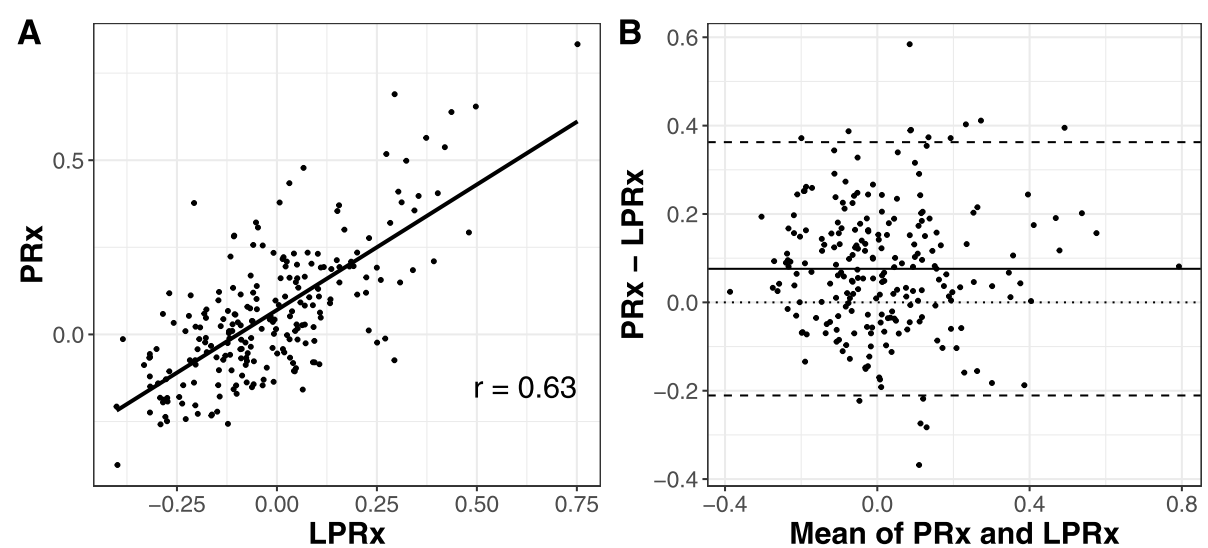

Fig. 1 a Spearman's correlation between LPRx and PRx. b Bland-Altman plot for agreement between PRx and LPRx showing mean bias (solid line), zero difference (thin dashed line), and 95\% lines of agreement (thick dashed lines)

analysis were similar when patients with decompressive surgery were excluded (data not shown).

\section{Optimal cerebral perfusion pressure}

Both the LPRx-based and the PRx-based CPPopt calculations produced a similar median CPPopt value in our patient cohort (71.4 (65.9-76.6) $\mathrm{mmHg}$ vs. 72.0 (65.977.5) $\mathrm{mmHg}, p=0.445$ ). Correspondingly, we found no significant difference in CPPopt yield between the LPRxand PRx-based calculations (80.0 (70.4-86.7) \% vs. 80.4 (71.4-87.6) \%, $p=0.625)$. The difference between CPP and $C_{P P o p t}{ }_{P R x}\left(\triangle C P P_{0 p t} t_{P R x}\right)$ was significantly higher in patients with fatal outcome compared to survivors (3.7 (1.9-5.8) $\mathrm{mmHg}$ vs. 1.9 (0.9-4.1) $\mathrm{mmHg}, p=0.003)$. This relation was also apparent when examining the $\triangle$ CPPopt $_{\mathrm{LPRx}}$, although it was considerably lower (2.3
(1.0-3.6) $\mathrm{mmHg} \quad$ vs. $1.5 \quad(0.7-2.9), \quad p=0.049)$. $\triangle$ CPPopt $_{\mathrm{LPRx}}$ and $\triangle \mathrm{CPPopt}_{\mathrm{PRx}}$ were significant predictors of mortality both in univariate and multivariate analyses with age, GCS-Motor, and pupillary as covariates, underlining the association between both CPPopt versions and clinical outcome (Table 2 (B)). However, when also including ICP and CPP in the multivariate model, only $\triangle$ CPPopt $_{\mathrm{PRx}}$ remained a significant predictor (Table 2 (D)). Still, there was no significant difference between the AUCs of $\triangle$ CPPopt $_{\mathrm{LPRx}}$ and $\triangle$ CPPopt $_{\text {PRx }}$ when predicting mortality $(0.67(0.59-0.76)$ $\Delta$ CPPopt $_{\text {LPRx }}$ vs. $0.71(0.62-0.79) \Delta$ CPPopt $_{\text {PRx }} ; p=0.237$; Fig. 3c). Assessment of the discriminative value of both $\triangle C P$ Popt variables over the post-traumatic course did reveal neither a clear increase/decrease over time nor a clear superiority of one $\triangle \mathrm{CPP}$ Popt variable over the other.
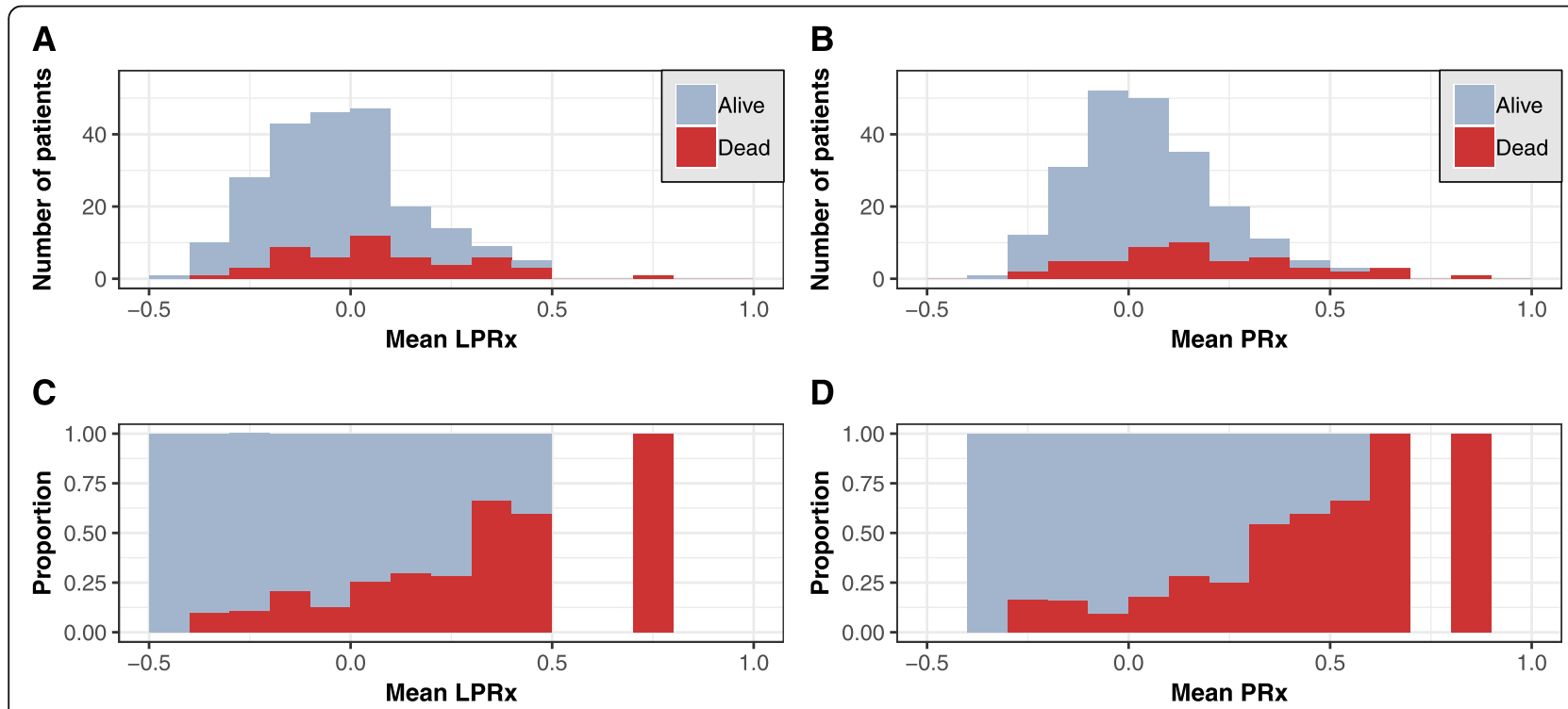

D

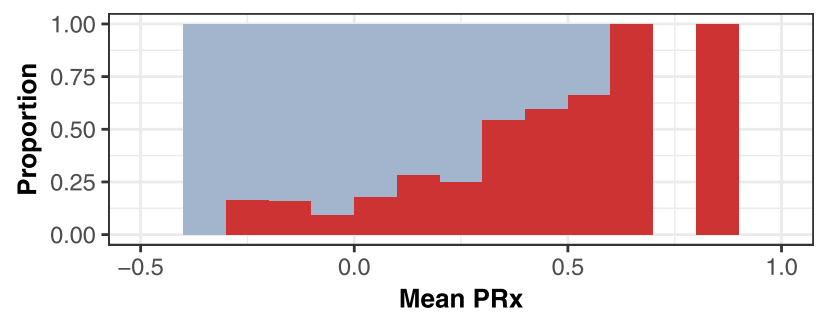

Fig. 2 Histograms showing the absolute $(\mathbf{a}, \mathbf{b})$ and relative $(\mathbf{c}, \mathbf{d})$ proportion of patients with fatal outcome for different LPRx and PRx values 
Table 2 Results from univariate and multivariate logistic regression analysis in regard to mortality for LPRx, PRx, $\triangle L P R x-C P P o p t$, and $\triangle \mathrm{PRx}$-CPPopt

\begin{tabular}{|c|c|c|c|c|c|}
\hline A & Parameter & Index: LPRx & & Index: PRx & \\
\hline & & Coefficient & $p$ value & Coefficient & $p$ value \\
\hline Univariate & Index & 3.484 & $<0.001$ & 4.244 & $<0.001$ \\
\hline Multivariate & Age & 0.039 & 0.001 & 0.036 & 0.003 \\
\hline & GCS-Motor & -0.191 & 0.068 & -0.202 & 0.059 \\
\hline & Pupillary response & 0.304 & 0.197 & 0.333 & 0.173 \\
\hline & Index & 2.634 & 0.011 & 3.868 & $<0.001$ \\
\hline B & Parameter & $\triangle$ CPPopt: LPRx & & $\triangle$ CPPopt: PRx & \\
\hline & & Coefficient & $p$ value & Coefficient & $p$ value \\
\hline Univariate & $\triangle C P P o p t$ & -0.122 & 0.003 & -0.148 & $<0.001$ \\
\hline Multivariate & Age & 0.049 & $<0.001$ & 0.050 & $<0.001$ \\
\hline & GCS-Motor & -0.196 & 0.059 & -0.227 & 0.034 \\
\hline & Pupillary response & 0.308 & 0.198 & 0.257 & 0.296 \\
\hline & $\triangle C P P o p t$ & -0.139 & 0.003 & -0.159 & $<0.001$ \\
\hline C & Parameter & Index: LPRx & & Index: PRx & \\
\hline & & Coefficient & $p$ value & Coefficient & $p$ value \\
\hline Univariate & Index & 3.484 & $<0.001$ & 4.244 & $<0.001$ \\
\hline Multivariate & Age & 0.043 & $<0.001$ & 0.039 & 0.002 \\
\hline & GCS-Motor & -0.164 & 0.130 & -0.169 & 0.127 \\
\hline & Pupillary response & 0.457 & 0.067 & 0.474 & 0.068 \\
\hline & $I C P$ & 0.071 & 0.005 & 0.061 & 0.019 \\
\hline & $C P P$ & 0.034 & 0.128 & 0.035 & 0.128 \\
\hline & Index & 1.628 & 0.152 & 3.140 & 0.007 \\
\hline D & Parameter & $\triangle$ CPPopt: LPRx & & $\Delta$ CPPopt: PRx & \\
\hline & & Coefficient & $p$ value & Coefficient & $p$ value \\
\hline Univariate & $\triangle C P P o p t$ & -0.122 & 0.003 & -0.148 & $<0.001$ \\
\hline Multivariate & Age & 0.050 & $<0.001$ & 0.051 & $<0.001$ \\
\hline & GCS-Motor & -0.166 & 0.124 & -0.190 & 0.086 \\
\hline & Pupillary response & 0.466 & 0.067 & 0.423 & 0.106 \\
\hline & $I C P$ & 0.067 & 0.009 & 0.065 & 0.011 \\
\hline & $C P P$ & 0.042 & 0.066 & 0.046 & 0.048 \\
\hline & Index & -0.108 & 0.065 & -0.146 & 0.002 \\
\hline
\end{tabular}

$\triangle C P P o p t$ difference between cerebral perfusion pressure and calculated optimal cerebral perfusion pressure, GCS-Motor Glasgow Coma Scale motor component, $L P R x$ long pressure reactivity index, PRx pressure reactivity index

However, a significant statistical difference could be observed at day 6 , with $\triangle$ CPPopt $_{\mathrm{PRx}}$ displaying a significant higher AUC compared to $\triangle C P P o p t L_{P R x}$ when predicting death ( $p=0.007$, DeLong's test; Fig. 4c, d). Similar to our findings with the LPRx and PRx, the goodness-of-fit was significantly improved when adding either $\triangle$ CPPopt $_{\mathrm{LPRx}}$ or $\triangle$ CPPopt $_{\mathrm{PRx}}$ to the basic IMPACT model $\left(\chi^{2}=9.39\right.$, $p=0.002\left(\right.$ IMPACT $+\Delta$ CPPopt $_{\text {LPRx }}$ ), and $\chi^{2}=16.71, p<$ 0.001 (IMPACT $+\Delta$ CPPopt $_{\text {PRx }}$ ); Fig. $3 \mathrm{~d}$ ). The LPRx plus IMPACT model displayed an AUC of 0.77 (0.69-0.85), and the PRx plus IMPACT model an AUC of 0.80 $(0.72-0.87)$. Similar to our previous findings with the
PRx and LPRx, equivalent results were obtained for all CPPopt-associated analyses without including surgically decompressed patients, except for results from the multivariate models including ICP and CPP where $\triangle$ CPPopt $_{\text {LPRX }}$ remained a significant predictor when only including patients without decompressive craniectomy (data not shown).

\section{Cerebral perfusion pressure targeted therapy}

Because a difference of $\pm 5 \mathrm{mmHg}$ between the actual CPP and calculated CPPopt has been proposed as an acceptable range for CPP-targeted therapy $[12,16]$, we 

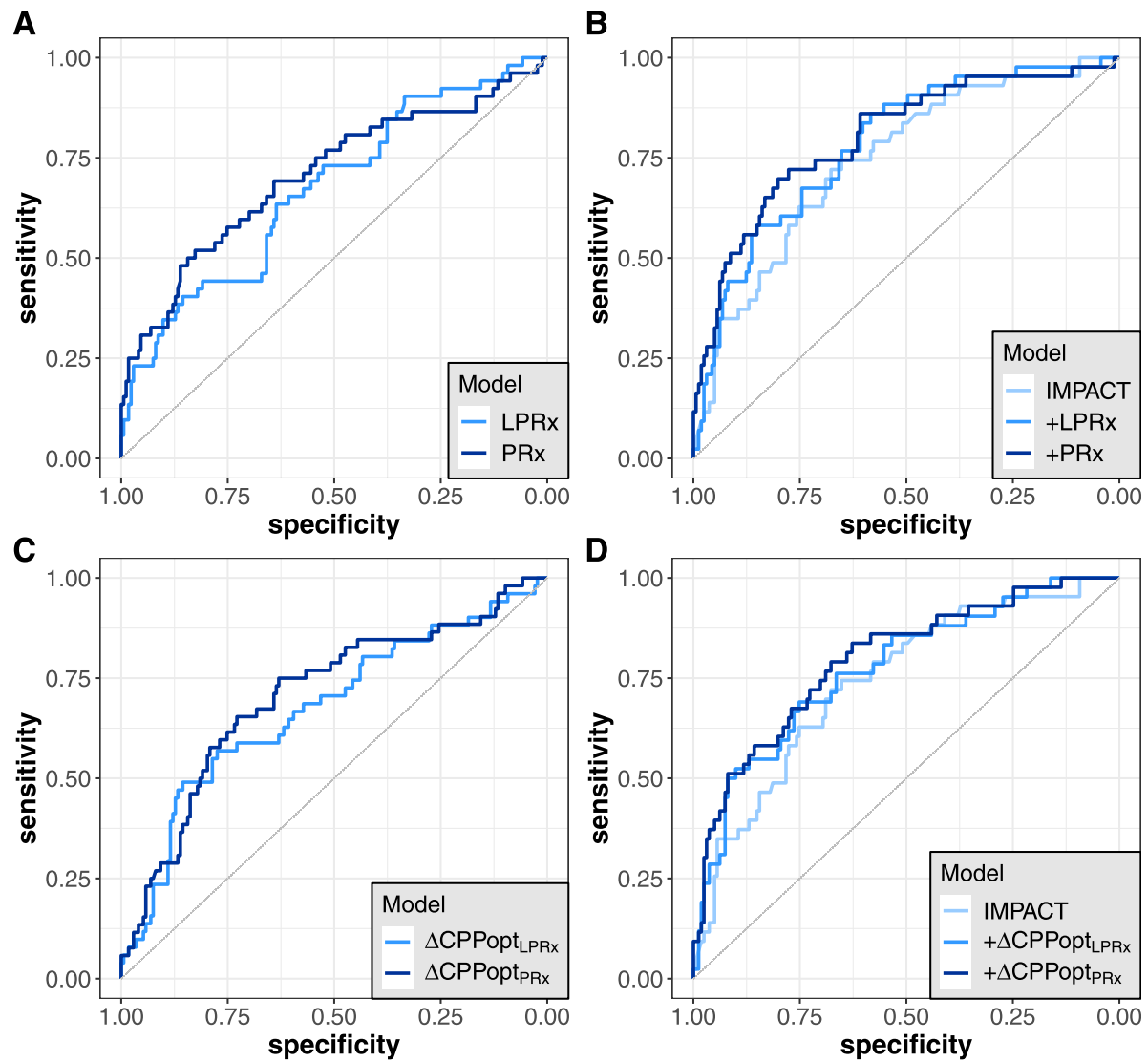

Fig. 3 AUCs for the prediction of mortality for different regression models. a LPRX and PRx. $\mathbf{b}$ IMPACT variables with the addition of LPRX and

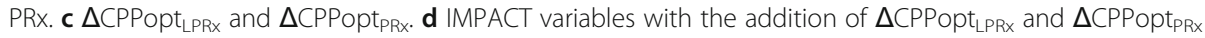

analyzed outcome in a subgroup of patients whose CPP deviated on average by at least $\pm 5 \mathrm{mmHg}$ from their calculated CPPopt LPRx $(n=32)$ and CPPopt PRx $(n=50$, Fig. $5 \mathrm{a}-\mathrm{d}$ ). Of 18 patients with a mean $\Delta \mathrm{CPPopt}_{\mathrm{PRx}}>5$ $\mathrm{mmHg}$ ("hyperperfused" patients), only one patient died (6\%). In contrast, 15 of 32 patients with a mean $\triangle$ CPPopt $_{\mathrm{PRx}}<-5 \mathrm{mmHg}$ ("hypoperfused" patients) were dead at the 6-month follow-up (47\%). The mortality rates differed significantly between those groups $\left(\chi^{2}=\right.$ $7.24, p=0.007)$. According to the calculated CPPopt LPRx $_{\text {, }}$ a mean $\triangle$ CPPopt $_{\text {LPRx }}>5 \mathrm{mmHg}$ was present in 18 patients with the same mortality rate of $6 \%$. However, only 14 patients were determined to be on average at least 5 $\mathrm{mmHg}$ below their CPPopt $_{\text {LPRx }}\left(\Delta\right.$ CPPopt $_{\text {LPRx }}<-5$ $\mathrm{mmHg}$ ), and of those, 5 had died (35\%). The mortality rates when comparing "hypo- and hyperperfused" patients according to $\mathrm{CPPopt}_{\mathrm{LPRx}}$ missed statistical significance $\left(\chi^{2}=2.93, p=0.087\right)$.

Eight of 32 patients (25\%) with a mean $\Delta$ CPPopt $_{\mathrm{PRx}}<$ $-5 \mathrm{mmHg}$ were severely disabled after 6 months. This proportion was distinctively higher in patients with a mean $\triangle$ CPPopt $_{\mathrm{PRx}}>5 \mathrm{mmHg}$ with 10 of 18 patients (56\%), although the rates of severe disability between those two patient groups did not reach statistical significance $\left(x^{2}=3.44, p=0.064\right)$. Similar results were found for the CPPopt LPRx 3 of 14 patients $(27 \%)$ with a mean $\triangle$ CPPopt $_{\text {LPRx }}<-5 \mathrm{mmHg}$ and 10 of 18 patients $(56 \%)$ with a mean $\triangle$ CPPopt $_{\text {LPRx }}>5 \mathrm{mmHg}$ were severely disabled 6 months after injury $\left(\chi^{2}=2.52, p=0.113\right)$. Again, similar results were obtained in all statistical tests when only patients without decompressive surgery were considered (data not shown).

\section{Discussion}

To individualize therapy is a promising concept for possible reduction of mortality and unfavorable outcome in patients with TBI. Regulating CPP according to computed CPPopt recommendations derived from cerebrovascular reactivity indices might hereby play an important role. However, it remains unclear if highresolution data for PRx calculation is necessary to obtain relevant CPPopt values or if low-resolution, minute-byminute signals for LPRx calculation might suffice. This question is particularly important because using the LPRx would make the CPPopt concept available to a wider range of centers and thus patients. The first two 


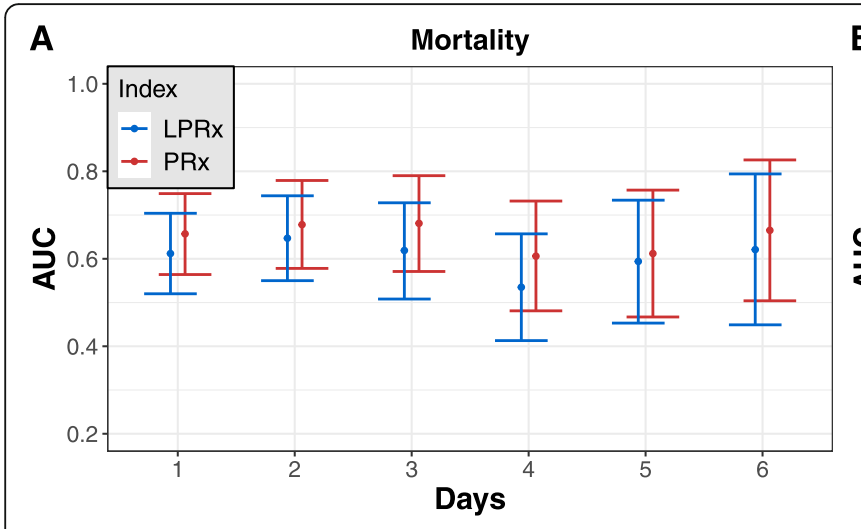

C

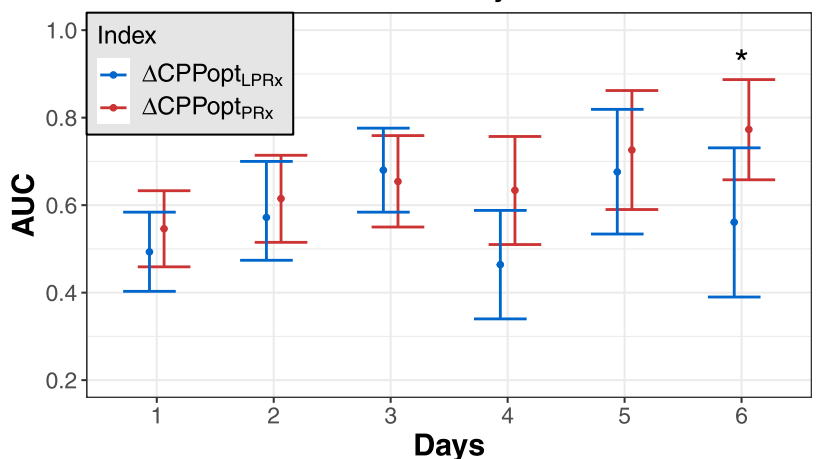

B

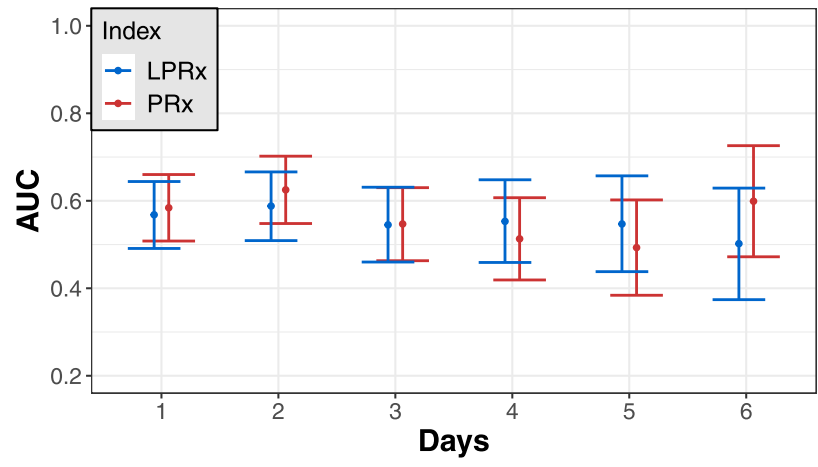

D

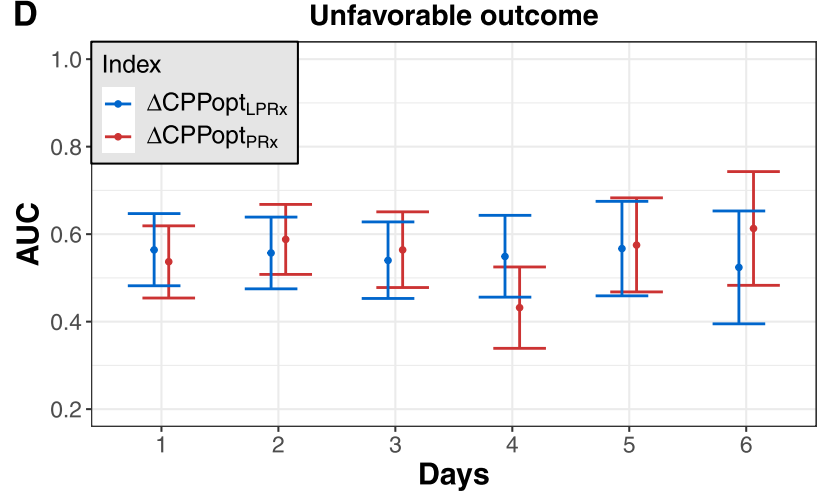

Fig. 4 AUCs for the prediction of mortality and unfavorable outcome over the early post-traumatic time course. a LPRx and PRx in regard to mortality. $\mathbf{b}$ LPRx and PRx in regard to unfavorable outcome. $\mathbf{c} \Delta$ CPPopt $_{L P R x}$ and $\Delta$ CPPopt $_{\text {PRx }}$ in regard to mortality. $\mathbf{d} \Delta$ CPPopt $_{\mathrm{LPRx}}$ and $\Delta$ CPPopt $_{\mathrm{PR}}$ in regard to unfavorable outcome. The only significant difference was observed at day 6 between LPRx and PRx $\left({ }^{*} p=0.007\right)$

pilot studies examining the LPRx showed encouraging results, stating that LPRx seemed to perform equally well in outcome prediction and CPPopt calculation as the PRx $[17,18]$. However, the validity of those studies was limited by relatively small patient numbers (18 and 29, respectively). More recently, a larger (over 300 patients) single-center follow-up study concluded the lowresolution LPRx to be less precise compared to PRx in outcome prediction which our results seem to confirm [8]. The proposed weaker discriminative ability could be further supported by our data especially when ICP and CPP were also added to the IMPACT variables in a multivariate model, where PRx but not LPRx remained a significant predictor. The reason for this inferior performance is likely that the LPRx only includes slower drifts in ABP and ICP which provide less information on the state of autoregulation, as opposed to the PRx which also includes higher frequency wave components, likely representing more outcome-relevant autoregulatory responses.

However, even when performing to some extent worse than the PRx in outcome prediction, the LPRx almost always showed significant results in our analyses as well and the differences of AUCs between PRx and LPRx in univariate regression to mortality were non-significant in our current work (albeit in a smaller sample). This was also true when comparing the discriminative value of both indices day-by-day during the early post-injury time course (first 6 days).

What is very important is that both indices significantly improved the performance of a multivariable model containing the IMPACT core variables and remained independent predictors for mortality after TBI. However, LPRx lost significance when also adjusting for ICP and CPP. Nevertheless, when taken together, those results seem to support the notion proposed by previous studies $[19,22]$ that minute-by-minute averaged signals, while performing to some extent weaker, might still carry important outcome-related information and might be sufficient for autoregulation monitoring via pressure reactivity indices. Notably, both indices performed considerably worse when predicting unfavorable outcome compared to predicting mortality, which is also in accordance with previous studies [10, 11]. A subgroup analysis showed an especially strong association with outcome for LPRx/PRx in patients with severe TBI, making our results particularly applicable for such patients.

Given the abovementioned findings, we sought to evaluate the performance of a weighted, multi-window 


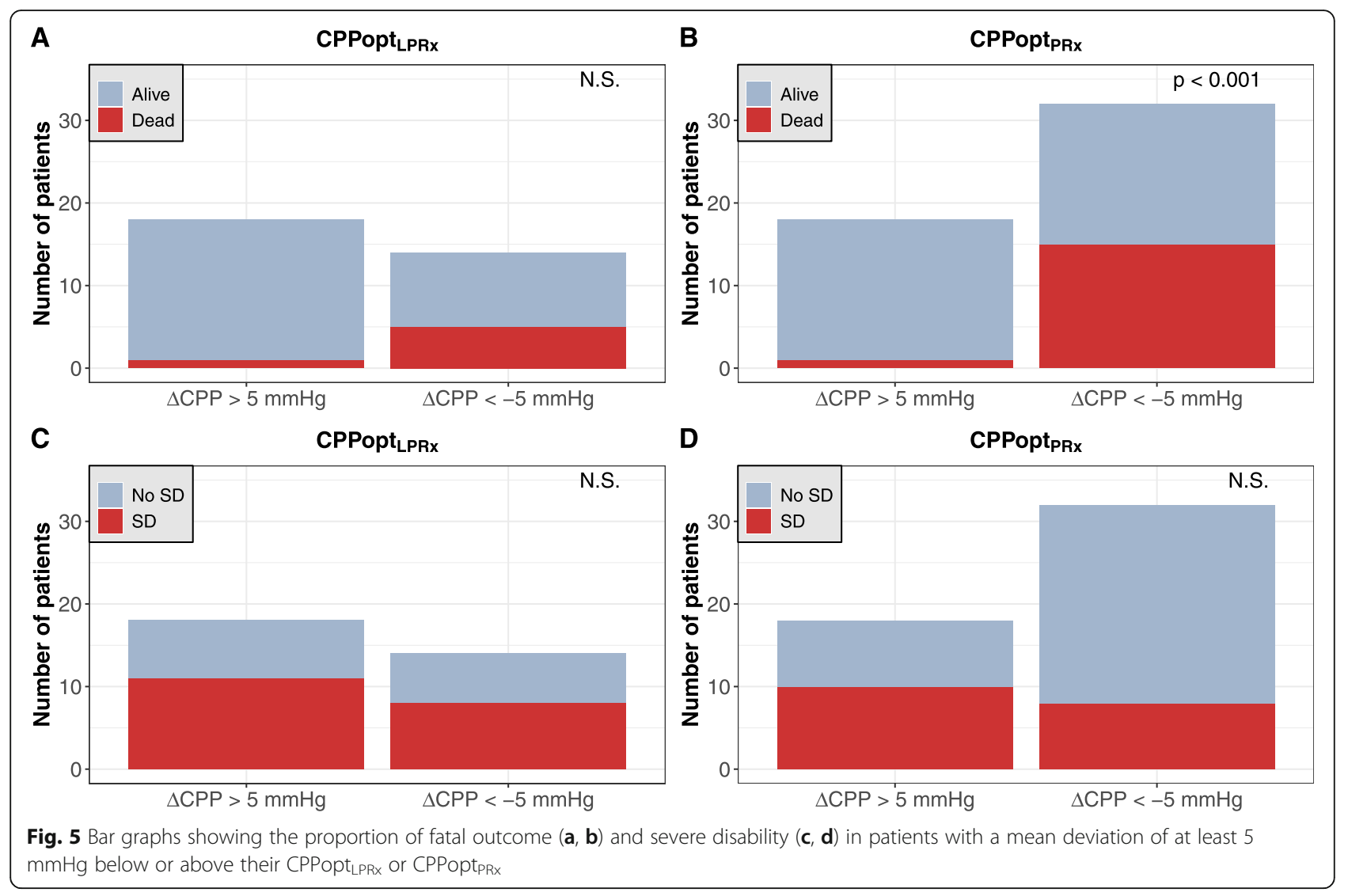

algorithm for assessing CPPopt that is based on the LPRx instead of the PRx. Determination of CPPopt has the potential to translate the PRx/LPRx concept into clinical management by offering dynamic management targets for CPP according to CPPopt. Ideally, patients might then directly benefit from this individualized therapy. The CPPopt concept built on PRx values has been shown to be of prognostic value in numerous studies in the sense that deviations of CPP from CPPopt were predictors of fatal outcome in TBI patients [12-15, 19, 23]. While the first automated CPPopt algorithm was based on PRx/CPP values in a moving single-window of $4 \mathrm{~h}$ for calculation [13], this method was extended to include multiple windows for calculations by Depreitere et al. [19], who used minute-by-minute monitoring data and various low-resolution indices for their approach. They could show that the resulting CPPopt was highly related to outcome and was not inferior in outcome prediction compared to the single-window CPPopt ${ }_{\mathrm{PRx}}$ which is based on high-resolution data.

The multi-window concept developed by Depreitere et al. was then adapted to high-resolution data, and thus $\mathrm{PRx}$, and extended with additional weighting and safety criteria as well as more calculation windows in an algorithm implemented in ICM+ by Liu et al. [14], and subsequently modified further to make it suitable for clinical, bedside application as part of the COGiTATE trial [16]. In our study, we sought to evaluate how the low-resolution LPRx, instead of the PRx, would perform in this most recent CPPopt calculation method. Using the CENTER-TBI high-resolution ICU cohort, we were able to do this in direct reference to the PRx-based approach and in a multi-center dataset. Similar to the LPRx itself, CPPopt $t_{L P R x}$ performed slightly worse in outcome prediction than its PRx counterpart but was still a significant predictor for mortality in univariate and multivariate analysis including the IMPACT variables. Both $\triangle$ CPPopt $_{\mathrm{LPRx}}$ and $\triangle$ CPPopt $_{\mathrm{PRx}}$ were significant predictors of mortality, even when patients were adjusted for other prognostic factors in multivariate analysis, and their addition to the IMPACT core model could significantly improve the goodness-of-fit. Addition of $\triangle \mathrm{CPPopt}$ displayed even higher AUCs for mortality than addition of reactivity indices to the model. Interestingly, when also including ICP and CPP in addition to the IMPACT variables in the model, $\triangle$ CPPopt $_{\mathrm{PRx}}$ remained significant while $\triangle$ CPPopt $_{\text {LPRx }}$ failed to demonstrate significance in the entire cohort. However, $\triangle C_{C P} P_{\text {opt }}{ }_{L P R x}$ remained significant in non-decompressed patients, indicating a potential use in this patient group. All those results seem to emphasize the importance that the deviation of CPP from CPPopt might have in regard to clinical outcome. 
Further studies should examine the extension of the IMPACT core model with autoregulation monitoring indices, analogous to the already present IMPACT core + CT model or IMPACT core + CT + laboratory markers model.

When only patients with a relevant mean CPP/CPPopt deviation were considered, the CPPopt $_{\mathrm{PRx}}$ method detected more patients overall and showed a closer relation to mortality in patients with an average deviation in CPP of at least $5 \mathrm{mmHg}$ below the PRx-CPPopt. Even the $\mathrm{CPPopt}_{\mathrm{LPRx}}$ showed a substantially higher mortality rate in "hypoperfused" patients when compared to "hyperperfused" ones, although this difference between mortality rates did not reach significance. Similar to Aries et al. [13], we found a higher rate of severe disability in "hyperperfused" patients compared to "hypoperfused" ones. However, this association did not reach significance in our study.

Concerning our results on CPPopt availability (yield of the algorithm), it has to be mentioned that CPPopt PRx $_{\text {I }}$ CPPopt $_{\text {LPRx }}$ could not be calculated in 8 patients, for reason not related to a failure of the CPPopt algorithm. In 5 patients, ICP was so high and CPP so low that the autoregulation was completely and entirely lost, and the concept of "optimal" CPP was therefore not applicable. In further 3 patients, there was simply not enough data available to perform the calculation. In all the remaining patients, the fraction of time where CPPopt could be determined was importantly very similar between CPPopt $_{\text {LPRx }}$ and CPPopt ${ }_{\text {PRx }}$.

Interestingly, the exclusion of patients who underwent decompressive surgery during their ICU stay did not affect the results in most of our analyses (data not shown), similar to previously published results in other studies. A notable exception is that $\triangle \mathrm{CPPopt}_{\mathrm{LPRx}}$ remained a significant predictor for mortality in a multivariate model including ICP and CPP only in nondecompressed patients while miss significance in the entire cohort. This is despite the fact that the performance of PRx and thus also LPRx, as the pressure reactivity monitor, depends on reliable transmission of changes in cerebral blood volume into intracranial pressure [24, 25], and that is theoretically adversely affected by the decompressive craniectomy. However, the exact timing of decompressive surgery could play an important role as indices are averaged over the whole monitoring period and future studies should be conducted to examine this relationship closer.

\section{Limitations}

As the CENTER-TBI study was designed to be a prospective observational study, treatment strategies and protocols in ICUs might considerably differ between participating centers and might therefore be confounders. Importantly,
ICP and mean arterial pressure (MAP) signals were subject to manipulation by treating clinicians (e.g., actively lowering ICP or MAP in selected patients) and might therefore be also the result of therapeutic interventions. Moreover, this study contains a heterogenous group of patients in terms of demographics, injury characteristics, and comorbidities which might influence the results. Finally, the considered variables such as the indices and differences between CPP and CPPopt were averaged over the entire monitoring time per patient which could mean that their variability and the presence of short periods with very deviated values were not accounted for in our analysis. Regarding the temporal course of the discriminative power of both indices, it is important to note that the sample size considerably decreased over time which might influence the results especially at later time points. While this multi-center study can provide evidence for the relevance of LPRx and CPPopt ${ }_{\mathrm{LPR} x}$, a high-quality, prospective study is needed to conclude whether the CPPopt $t_{\mathrm{LPRx}}$ concept can be translated into clinical benefit in patients with TBI.

\section{Conclusions}

Our findings indicate that the LPRx and multi-window CPPopt $_{\mathrm{LPRx}}$ do not reach the precision of the PRx and CPPopt $_{\mathrm{PRx}}$ in outcome prediction after TBI. However, the LPRx is still significantly associated with outcome and can produce outcome-relevant CPPopt values, most reliably in non-decompressed patients. A prospective trial is needed to assess if this association is strong enough for a meaningful clinical translation. Should the ongoing COGiTATE feasibility/safety trial and subsequent phase 3 trials be successful in proving the CPPopt concept, it might be worthy to consider a respective trial with the CPPopt ${ }_{\mathrm{LPRx}}$ to make the CPPopt concept available to a broader range of centers and patients.

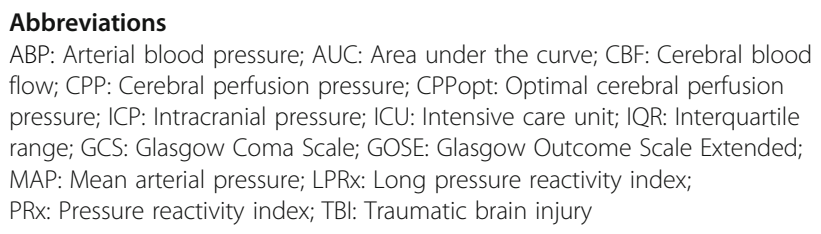

\section{Acknowledgements}

The names of the individual members of the CENTER-TBI High Resolution ICU (HR ICU) Sub-Study Participants and Investigators collaboration group who helped gathering the data for this manuscript should be searchable through their individual PubMed records. Furthermore, we gratefully acknowledge the support from all CENTER-TBI investigators and participants and we are thankful to all participating patients for helping us to improve clinical care and outcome after TBI. Finally, we thank Julia Mattern and Madlen Rädel for their help withthe local organization of the CENTER-TBI study at Heidelberg University Hospital.

CENTER-TBI High Resolution ICU (HR ICU) Sub-Study Participants and Investigators: Audny Anke, Department of Physical Medicine and Rehabilitation, University Hospital Northern Norway; Ronny Beer, Department of Neurology, Neurological Intensive Care Unit, Medical University of Innsbruck, Innsbruck, Austria; Bo-Michael Bellander, Department of Neurosurgery \& Anesthesia \& 
Intensive Care Medicine, Karolinska University Hospital, Stockholm, Sweden; Andras Buki, Department of Neurosurgery, University of Pecs and MTA-PTE Clinical Neuroscience MR Research Group and Janos Szentagothai Research Centre, University of Pecs, Hungarian Brain Research Program, Pecs, Hungary; Giorgio Chevallard, Neurolntensive Care, Niguarda Hospital, Milan, Italy; Arturo Chieregato, Neurolntensive Care, Niguarda Hospital, Milan, Italy; Giuseppe Citerio, Neurolntensive Care Unit, Department of Anesthesia \& Intensive Care, ASST di Monza, Monza, Italy; and School of Medicine and Surgery Università Milano Bicocca, Milano, Italy; Endre Czeiter, Department of Neurosurgery, University of Pecs and MTA-PTE Clinical Neuroscience MR Research Group and Janos Szentagothai Research Centre, University of Pecs, Hungarian Brain Research Program (Grant No. KTIA 13 NAP-A-II/8), Pecs, Hungary; Bart Depreitere, Department of Neurosurgery, University Hospitals Leuven, Leuven, Belgium; George Eapen t, Shirin Frisvold, Department of Anesthesiology and Intensive Care, University Hospital Northern Norway, Tromso, Norway; Raimund Helbok, Department of Neurology, Neurological Intensive Care Unit, Medical University of Innsbruck, Innsbruck, Austria; Stefan Jankowski, Neurointensive Care, Sheffield Teaching Hospitals NHS Foundation Trust, Sheffield, UK; Daniel Kondziella, Departments of Neurology, Clinical Neurophysiology and Neuroanesthesiology, Region Hovedstaden Rigshospitalet, Copenhagen, Denmark; Lars-Owe Koskinen, Department of Clinical Neuroscience, Neurosurgery, Umea University Hospital, Umea, Sweden; Geert Meyfroidt, Intensive Care Medicine, University Hospitals Leuven, Leuven, Belgium; Kirsten Moeller, Department Neuroanesthesiology, Region Hovedstaden Rigshospitalet, Copenhagen, Denmark; David Nelson, Department of Neurosurgery \& Anesthesia \& intensive care medicine, Karolinska University Hospital, Stockholm, Sweden; Anna Piippo-Karjalainen, Helsinki University Central Hospital, Helsinki, Finland; Andreea Radoi, Department of Neurosurgery, Vall d'Hebron University Hospital, Barcelona, Spain; Arminas Ragauskas, Department of Neurosurgery, Kaunas University of technology and Vilnius University, Vilnius, Lithuania; Rahul Raj, Helsinki University Central Hospital, Helsinki, Finland; Jonathan Rhodes, Department of Anaesthesia, Critical Care \& Pain Medicine NHS Lothian \& University of Edinburg, Edinburgh, UK; Saulius Rocka, Department of Neurosurgery, Kaunas University of technology and Vilnius University, Vilnius, Lithuania; Rolf Rossaint, Department of Anaesthesiology, University Hospital of Aachen, Aachen, Germany; Juan Sahuquillo, Department of Neurosurgery, Vall d'Hebron University Hospital, Barcelona, Spain; Ana Stevanovic, Department of Anaesthesiology, University Hospital of Aachen, Aachen, Germany; Nina Sundström, Department of Radiation Sciences, Biomedical Engineering, Umea University Hospital, Umea, Sweden; Riikka Takala, Perioperative Services, Intensive Care Medicine, and Pain Management, Turku University Central Hospital and University of Turku, Turku, Finland; Tomas Tamosuitis, Neuro-intensive Care Unit, Kaunas University of Health Sciences, Kaunas, Lithuania; Olli Tenovuo, Rehabilitation and Brain Trauma, Turku University Central Hospital and University of Turku, Turku, Finland; Peter Vajkoczy, Neurologie, Neurochirurgie und Psychiatrie, CharitéUniversitätsmedizin Berlin, Berlin, Germany; Alessia Vargiolu, Neurolntensive Care Unit, Department of Anesthesia \& Intensive Care, ASST di Monza, Monza, Italy; Rimantas Vilcinis, Department of Neurosurgery, Kaunas University of Health Sciences, Kaunas, Lithuania; Stefan Wolf, Interdisciplinary Neuro Intensive Care Unit, Charité-Universitätsmedizin Berlin, Berlin, Germany.

\section{Authors' contributions}

Study design: $L R, E B, P S$, and $A Y$; ICM+ implementation and algorithm: $L R$ $E B, P S$, and $M C$; data analysis: $L R$ and $A Y$; data interpretation: $L R, E B, P S, M C$, $N S, O S, K Z, A U$, and $A Y$; writing the manuscript: $L R$ and $A Y$; reviewing and editing the manuscript: LR, EB, PS, MC, NS, OS, KZ, AU, and AY. All authors read and approved the final manuscript.

\section{Funding}

CENTER-TBI was supported by the European Union 7th Framework program (EC grant 602150). Additional funding was obtained from the Hannelore Kohl Stiftung (Germany), OneMind (USA), and Integra LifeSciences Corporation (USA).

\section{Availability of data and materials}

Data from the CENTER-TBI study is available to researchers after submitting a detailed study proposal (https://www.center-tbi.eu/data.) that is approved by the CENTER-TBI management committee. Data from this study is available from the corresponding author upon reasonable request and with permission of the CENTER-TBI management committee.

\section{Ethics approval and consent to participate}

The CENTER-TBI study (EC grant 602150) is compliant with all relevant EU and national laws of recruiting centers in regard to privacy, data protection, and ethical standards and in accordance with the Declaration of Helsinki ("Ethical Principles for Medical Research Involving Human Subjects.") Informed consent was obtained from all patients and/or their legal representatives, according to the local legislations, included in this study. Ethical approval was obtained for each recruiting site (see https://www.center-tbi. eu/project/ethical-approval for the list of sites, ethical committees, approval numbers, and approval dates)

\section{Consent for publication}

Not applicable.

\section{Competing interests}

LR received a scholarship from the CENTER-TBI study to visit the Brain Physics Lab in Cambridge. MC and PS receive part of the licensing fee for the ICM+ software. MC is supported by the NIHR Biomedical Research Centre Cambridge. The remaining authors have nothing to disclose.

\section{Author details}

${ }^{1}$ Department of Neurosurgery, Heidelberg University Hospital, INF 400, 69120 Heidelberg, Germany. ${ }^{2}$ Brain Physics Laboratory, Division of Neurosurgery, Department of Clinical Neurosciences, University of Cambridge, Cambridge, UK. ${ }^{3}$ Department of Physiopathology and Transplantation, Milan University, Milan, Italy. ${ }^{4}$ Neuro ICU Fondazione IRCCS Cà Granda Ospedale Maggiore Policlinico, Milan, Italy. ${ }^{5}$ Department of Neurosurgery, Klinikum Ludwigsburg, Ludwigsburg, Germany.

Received: 17 December 2019 Accepted: 12 May 2020

Published online: 26 May 2020

\section{References}

1. Rangel-Castilla L, Gasco J, Nauta HJW, Okonkwo DO, Robertson CS. Cerebral pressure autoregulation in traumatic brain injury. Neurosurg Focus. 2008;25: E7 [cited 2019 May 1]. Available from: http://www.ncbi.nlm.nih.gov/ pubmed/18828705.

2. Kelly DF, Martin NA, Kordestani R, Counelis G, Hovda DA, Bergsneider M, et al. Cerebral blood flow as a predictor of outcome following traumatic brain injury. J Neurosurg. 1997;86:633-41 [cited 2019 Jul 2]. Available from: http://www.ncbi.nlm.nih.gov/pubmed/9120627.

3. Werner $\mathrm{C}$, Engelhard K. Pathophysiology of traumatic brain injury. Br J Anaesth. 2007;99:4-9 [cited 2019 Jul 2]. Available from: https://linkinghub. elsevier.com/retrieve/pii/S0007091217347840. Narnia.

4. Hlatky R, Valadka AB, Robertson CS. Intracranial pressure response to induced hypertension: role of dynamic pressure autoregulation. Neurosurgery. 2005;57:917-23 [cited 2019 Jul 6]. discussion 917-23. Available from: http://www.ncbi.n/m.nih.gov/pubmed/16284561.

5. Jünger EC, Newell DW, Grant GA, Avellino AM, Ghatan S, Douville CM, et al. Cerebral autoregulation following minor head injury. J Neurosurg. 1997;86: 425-32 [cited 2019 Jul 6]. Available from: http://www.ncbi.nlm.nih.gov/ pubmed/9046298.

6. Carney N, Totten AM, O'Reilly C, Ullman JS, Hawryluk GWJ, Bell MJ, et al. Guidelines for the management of severe traumatic brain injury, fourth edition. Neurosurgery. 2016;80:1 [cited 2019 Jul 2]. Available from: http:// www.ncbi.nlm.nih.gov/pubmed/27654000.

7. Robertson CS, Valadka AB, Hannay HJ, Contant CF, Gopinath SP, Cormio M, et al. Prevention of secondary ischemic insults after severe head injury. Crit Care Med. 1999;27:2086-95 [cited 2019 Jul 2]. Available from: http://www. ncbi.nlm.nih.gov/pubmed/10548187.

8. Lang EW, Kasprowicz M, Smielewski P, Santos E, Pickard J, Czosnyka M. Short pressure reactivity index versus long pressure reactivity index in the management of traumatic brain injury. J Neurosurg. 2015;122:588-94 [cited 2018 Sep 9]. Available from: http://thejns.org/doi/10.3171/2014.10.JNS14602. American Association of Neurological Surgeons.

9. Czosnyka M, Smielewski P, Kirkpatrick P, Laing RJ, Menon D, Pickard JD. Continuous assessment of the cerebral vasomotor reactivity in head injury. Neurosurgery. 1997;41:11-7 [cited 2019 May 1]. discussion 17-9. Available from: http://www.ncbi.nlm.nih.gov/pubmed/9218290.

10. Sorrentino E, Diedler J, Kasprowicz M, Budohoski KP, Haubrich C, Smielewski $P$, et al. Critical thresholds for cerebrovascular reactivity after traumatic brain 
injury. Neurocrit Care. 2012;16:258-66 [cited 2019 Apr 30]. Available from: http://link.springer.com/10.1007/s12028-011-9630-8. Humana Press Inc.

11. Zeiler FA, Ercole A, Cabeleira M, Zoerle T, Stocchetti N, Menon DK, et al. Univariate comparison of performance of different cerebrovascular reactivity indices for outcome association in adult TBI: a CENTER-TBI study. Acta Neurochir (Wien). 2019:1-11 [cited 2019 May 1]. Available from: http://link. springer.com/10.1007/s00701-019-03844-1. Springer Vienna.

12. Steiner LA, Czosnyka M, Piechnik SK, Smielewski P, Chatfield D, Menon DK, et al. Continuous monitoring of cerebrovascular pressure reactivity allows determination of optimal cerebral perfusion pressure in patients with traumatic brain injury. Crit Care Med. 2002;30 Available from: https:// journals.lww.com/ccmjournal/Fulltext/2002/04000/Continuous_monitoring_ of_cerebrovascular_pressure.2.aspx.

13. Aries MJH, Czosnyka M, Budohoski KP, Steiner LA, Lavinio A, Kolias AG, et al. Continuous determination of optimal cerebral perfusion pressure in traumatic brain injury. Crit Care Med. 2012;40:2456-63 [cited 2019 May 28]. Available from: http://www.ncbi.n/m.nih.gov/pubmed/22622398

14. Liu X, Maurits NM, Aries MJH, Czosnyka M, Ercole A, Donnelly J, et al. Monitoring of optimal cerebral perfusion pressure in traumatic brain injured patients using a multi-window weighting algorithm. J Neurotrauma. 2017; 34:3081-8 [cited 2019 Jun 13]. Available from: http://www.liebertpub.com/ doi/10.1089/neu.2017.5003. Mary Ann Liebert, Inc. 140 Huguenot Street, 3rd Floor New Rochelle, NY 10801 USA.

15. Zeiler FA, Ercole A, Cabeleira M, Carbonara M, Stocchetti N, Menon DK, et al. Comparison of performance of different optimal cerebral perfusion pressure parameters for outcome prediction in adult traumatic brain injury: a Collaborative European NeuroTrauma Effectiveness Research in Traumatic Brain Injury (CENTER-TBI) study. J Neurotrauma. 2019:36:1505-17 [cited 2019 Jun 24]. Available from: https:/www.liebertpub.com/doi/10.1089/neu.201 8.6182. Mary Ann Liebert, Inc., publishers 140 Huguenot Street, 3rd Floor New Rochelle, NY 10801 USA.

16. Beqiri E, Smielewski P, Robba C, Czosnyka M, Cabeleira MT, Tas J, et al. Feasibility of individualised severe traumatic brain injury management using an automated assessment of optimal cerebral perfusion pressure: the COGiTATE phase II study protocol. BMJ Open. 2019;9:e030727 [cited 2019 Nov 4]. Available from: http://www.ncbi.nlm.nih.gov/pubmed/31542757. British Medical Journal Publishing Group.

17. Santos E, Diedler J, Sykora M, Orakcioglu B, Kentar M, Czosnyka M, et al. Low-frequency sampling for PRx calculation does not reduce prognostication and produces similar CPPopt in intracerebral haemorrhage patients. Acta Neurochir (Wien). 2011;153:2189-95 [cited 2018 Aug 26]. Available from: http://ink.springer.com/10.1007/s00701-011-1148-5. Springer Vienna.

18. Sánchez-Porras R, Santos E, Czosnyka M, Zheng Z, Unterberg AW, Sakowitz $\mathrm{OW}$. 'Long' pressure reactivity index (L-PRx) as a measure of autoregulation correlates with outcome in traumatic brain injury patients. Acta Neurochir (Wien). 2012;154:1575-81 [cited 2018 Aug 26]. Available from: http://link. springer.com/10.1007/s00701-012-1423-0. Springer Vienna.

19. Depreitere B, Güiza F, Van den Berghe G, Schuhmann MU, Maier G, Piper I, et al. Pressure autoregulation monitoring and cerebral perfusion pressure target recommendation in patients with severe traumatic brain injury based on minute-by-minute monitoring data. J Neurosurg. 2014;120:1451-7 [cited 2019 Jul 3]. Available from: http://www.ncbi.nlm.nih.gov/pubmed/24745709.

20. Steyerberg EW, Mushkudiani N, Perel P, Butcher I, Lu J, McHugh GS, et al. Predicting outcome after traumatic brain injury: development and international validation of prognostic scores based on admission characteristics. PLoS Med. 2008;5:e165 [cited 2019 Jul 31]. Available from: http://www.ncbi.nlm.nih.gov/pubmed/18684008. Singer M, editor.

21. R Core Team. R: A Language and Environment for Statistical Computing [Internet]. Vienna, Austria; 2018. Available from: https://www.r-project.org/.

22. Güiza F, Depreitere B, Piper I, Van den Berghe G, Meyfroidt G. Novel methods to predict increased intracranial pressure during intensive care and long-term neurologic outcome after traumatic brain injury. Crit Care Med. 2013;41:554-64 [cited 2019 Jul 6]. Available from: http://www.ncbi.nlm. nih.gov/pubmed/23263587.

23. Zweifel C, Lavinio A, Steiner LA, Radolovich D, Smielewski P, Timofeev I, et al. Continuous monitoring of cerebrovascular pressure reactivity in patients with head injury. Neurosurg Focus. 2008;25:E2 [cited 2019 May 3]. Available from: http://www.ncbi.nlm.nih.gov/pubmed/18828700

24. Aries MJH, Czosnyka M, Budohoski KP, Kolias AG, Radolovich DK, Lavinio A, et al. Continuous monitoring of cerebrovascular reactivity using pulse waveform of intracranial pressure. Neurocrit Care. 2012;17:67-76 [cited 2019 Nov 4]. Available from: http://ink.springer.com/10.1007/s12028-012-9687-z. Humana Press Inc.

25. Marmarou A, Shulman K, Rosende RM. A nonlinear analysis of the cerebrospinal fluid system and intracranial pressure dynamics. J Neurosurg. 1978:48:332-44 [cited 2019 Aug 8]. Available from: http://www.ncbinlm.nih. gov/pubmed/632857.

\section{Publisher's Note}

Springer Nature remains neutral with regard to jurisdictional claims in published maps and institutional affiliations.
Ready to submit your research? Choose BMC and benefit from:

- fast, convenient online submission

- thorough peer review by experienced researchers in your field

- rapid publication on acceptance

- support for research data, including large and complex data types

- gold Open Access which fosters wider collaboration and increased citations

- maximum visibility for your research: over $100 \mathrm{M}$ website views per year

At BMC, research is always in progress.

Learn more biomedcentral.com/submissions 